\title{
Physicians' Perspectives of a Therapeutic Conversion within a Staff-model HMO
}

\author{
Gina S. Gilomen-Study, \\ J. Warren Salmon, T.K. Shea, and J.G. Frick
}

\section{OBJECTIVE:}

To identify perspectives of primary care physicians in a staff-model health maintenance organization (HMO) concerning a therapeutic conversion program conducted for the drug class HMG-CoA reductase inhibitors.

\section{DESIGN:}

Multi-site, observational study.

\section{SETTING:}

11 ambulatory care medical practices within a staff-model HMO serving 100,000 patients in the midwestern United States.

\section{PARTICIPANTS:}

25 adult medicine physicians specializing in internal medicine or family practice.

\begin{abstract}
MAIN OUTCOME MEASURES:
Physicians' perspectives of 13 components of the Lescol (fluvastatin) therapeutic conversion program as measured by a Likerttype scale.
\end{abstract}

\section{RESULTS:}

Means were used to quantify the overall response for each item. Findings indicated that the program rationale was clear, the supporting communications were useful, the inconveniences were slight, the patient feedback was neutral, and, on average, any clinical concerns about Lescol were insignificant.

\section{CONCLUSION:}

The responding physicians were not displeased with the Lescol therapeutic con- version program, which increased the prescribing of preferred and alternative agents from $20.6 \%$ within this class at the close of Q1 1994 to $69.2 \%$ at the close of Q1 1995 , resulting in a $26.5 \%$ reduction in average ingredient costs over the 12-month period. Nevertheless, the vast majority of physicians $(96 \%)$ strongly agreed that practicing physician opinion should be solicited prior to the implementation of future therapeutic conversion programs.

\section{KEY WORDS:}

Therapeutic conversion/substitution, Physician perspective, Formularies, Prescribing.

J Managed Care Pharm 1997; 3: 55-56; 65
The use of restrictive formularies within health maintenance organizations (HMOs) and the profiling of physicianprescribing patterns may limit practitioners' choices of drug therapy.' With more stringent utilization-management strategies, physicians now are finding themselves subject to unprecedented efforts to constrain the fiscal impact of their patient-care decisions. In particular, the computerization of medical records has facilitated sophisticated tracking techniques focused on individual physician practice profiles. ${ }^{2}$ While such tracking systems are essential tools for most managed care pharmacy administrators, their characterization as "big brother" or "the pharmacy police" should be avoided in settings where collaborative relationships between pharmacists and physicians are being sought.

With this in mind, administrative personnel at the staff-model HMO under study implemented a therapeutic conversion program that met the goals of a professional commitment to quality care while greatly lowering costs within the HMG-CoA reductase inhibitors drug class

\section{METHODS}

\section{Practice Settings}

This evaluation of physicians' perspectives was conducted at a staff-model HMO serving more than 100,000 members (95\% commercial enrollment, 5\% Medicare, and $0 \%$ Medicaid) in northeastern Illinois and northern Indiana. At the time of this study, the ambulatory physician practices consisted of 11 medical offices located throughout the metropolitan area. A total of 80 physicians (adult medicine and pediatrics) were employed by the HMO to practice within the 11 facilities.

\section{A Authors}

GINA S. GILOMEN-STUDY, PhARM.D., PH.D (candidate), is Managed Care Pharmacy Resident and doctoral student in Pharmacy Administration; J.W. SALMON, PH.D., is Professor and Director of the Managed Care Pharmacy Residency; and T.K. SHEA, PHARM.D., and J.G. FRICK, R.PH., M.S. (candidate), are residency preceptors with the Department of Pharmacy Administration, College of Pharmacy, University of Illinois at Chicago.

Reprints: Gina S. Gilomen-Study, College of Pharmacy, University of Illinois at Chicago, 833 South Wood Street, M/C 871, Chicago, IL 60612-7231.

Paper presented at: Academy of Managed Care Pharmacy Eighth Annual Meeting, San Francisco, CA, May 1996.

Copyright $(\mathcal{O}$ 1997, Acaclemy of Managed Care Pharmacy, Inc. All rights reserved. 
Except for the drug classes of HMG-CoA reductase inhibitors, $\mathrm{H} 2$ antagonists, and oral contraceptives, physicians within this setting use an otherwise open formulary. An internal pharmacy and therapeutics committee, consisting of nine physicians and three pharmacists, makes decisions regarding the content of the formulary. The open portion of the formulary is no more than a list of preferred drugs from which the physician is encouraged to prescribe. The three "restricted" classes of drugs on the formulary limit coverage to formulary drugs. A prior authorization process must be completed to obtain coverage for nonformulary products within the restricted classes of the formulary. This involves completion of a written prior authorization form by the physician who is required to document the medical justification for requesting a nonformulary product. This justification for the given case then is reviewed by the director of pharmacy and/or the associate medical director to determine clinical necessity. Physician profiling of preferred formulary products is conducted to encourage formulary adherence and to monitor the progress of the conversion process.

In January 1995, this staff-model HMO initiated a therapeutic conversion program for the drug class HMG-CoA reductase inhibitors. The antilipidemic agent Lescol (fluvastatin) was selected as the preferred product for use in primary prevention patients. Kellick ${ }^{3}$ has reported that $60 \%$ of primary prevention patients reached their cholesterol reduction goal with fluvastatin $20 \mathrm{mg}$. Pravachol (pravastatin) was selected as the alternative product 10 be prescribed in cases in which greater than a $30 \%$ reduction in cholesterol was necessitated and/or for secondary prevention patients with known coronary artery disease (CAD). At that time, Mevacor (lovastatin) and Zocor (simvastatin) were removed from the formulary, and physicians were notified to switch patients currently receiving these antilipidemic agents to the new preferred or alternative product.

Internal upgrades in the data retrieval systems made it possible to efficiently target communications and track progress within the 11 medical offices. Where the prior therapeutic conversion program was limited to memorandum notifications to all HMO physicians, improvements in the drug-use evaluation process allowed for a more sophisticated intervention with the antilipidemic agents. Several written communications were sent to the HMO physicians to facilitate this therapeutic conversion.

Physician letters, sent in January 1.995, explained the rationale behind this therapeutic conversion, presenting it as an opportunity to lower costs for antilipidemic drug therapy while maintaining clinical efficacy. The letter also explained how, by restricting the drug class HMG-CoA reductase inhibitors from four products to two, savings could be realized without sacrificing quality.

To facilitate the process, each physician also received lists of patients within each practice site affected by this conversion. Follow-up letters sent in February 1995 reminded physicians of the conversion and offered assistance in the refill-authorization communications between a physician's office and the patient's pharmacy.
To prepare members for the conversion, patients receiving prescriptions for Mevacor or Zocor were notified early in January 1995 via a letter and with a list of commonly asked questions and answers. As an incentive, the prescription copayments were waived for the first three months following the conversion from Mevacor or Zocor to Lescol or Pravachol.

Six months into the Lescol conversion program, an evaluation was requested to assess physicians' perspectives based on their participation and experiences with patients affected by the conversion. This presented an opportunity to consider the qualitative aspects of a therapeutic conversion as well as the new quantitative capabilities made possible through the improved data-retrieval system.

\section{Research Intervention}

A Likert-lype questionnaire was developed by interviewing several practicing HMO physicians and extracting their most salient concerns about this therapeutic conversion program. An 11-point Likert-type scale was used for each statement, with anchors of 0 ("highly disagree") and 10 ("highly agree"). The 13-item questionnaire was piloted and revised before being forwarded to 70 adult medicine physicians within the multi-site HMO system. Physicians were asked to respond within 10 days.

\section{RESULTS}

A response rate of $35.7 \%$ ( 25 of 70 ) was achieved by the 10-day cut-off set for this questionnaire. Rankings for each of the 13 items were analyzed using Systat 5.0 for Windows. Means and standard deviations were used to quantify the overall response for each of the 13 items.

\section{Rationale}

For the statement, "I clearly understood the rationale of the HMG-CoA reductase therapeutic conversion program," a mean (S.D.) response of $8.560+2.083$ was generated. These results showed that responding physicians agreed that the rationale of this therapeutic conversion was understood.

\section{Supporting Communications}

For the statement, "The supporting communications provided by pharmacy administration were useful to me," a mean response of $7.160+2.897$ was generated in reference to staff meetings, and a mean response of $8.000+1.708$ was generated in reference to written communications. These results indicated that responding physicians agreed that staff meetings and written communications were useful in this therapeutic conversion program.

\section{Clinical Concerns}

Three items were used to evaluate the three types of clinical concerns: short-term efficacy of lipid reduction, side effects, Continued on page 65 
and long-term effect on cardiovascular morbidity and mortality. For the statement, "I have clinical concerns related to this therapeutic conversion program," a mean response of $5.060+$ 3.336 was generated for short-term efficacy; a mean response of $3.940+3.235$ was generated for side effects; and a mean response of $4.820+3.294$ was generated for long-term effects. A bimodal distribution indicated that responding physicians were equally split in their clinical concerns regarding the short-term efficacy of lipid reduction. The frequency distribution for clinical concerns regarding side effects indicated that a greater proportion of responding physicians had few to no concerns with Lescol's side effects. Lastly, the frequency distribution for longterm effects indicated that a sizable proportion of responding physicians expressed no opinion about long-term effects on cardiovascular morbidity and mortality.

\section{Inconvenience}

Three items were used to evaluate the three types of inconveniences voiced during the physician interviews: increased paperwork, imposition on time, and credibility with patients. For the statement, "This therapeutic conversion program has been an inconvenience to my medical practice," a mean response rate of $6.040+2.606$ was generated for increased paperwork; $5.960+3.075$ for imposition on time; and $5.760+$ 2.570 for credibility with patients. These results showed that responding physicians slightly agreed that increased paperwork was an inconvenience with this therapeutic conversion. In general, they also slightly agreed that there was an inconvenience because of imposition on time and an inconvenience because of credibility issues with patients.

\section{Patient Feedback}

Three items were used to evaluate the three types of patient feedback voiced during the physician interviews: convenience receiving the prescription, patient perception of therapeutic response, and patient's willingness to switch products. For the statement, "The patient feedback that I have received has been positive," a mean response of $4.680+2.545$ was generated for convenience receiving the prescription; $4.640+1.777$ for patient perception of therapeutic response; and $5.400+2.345$ for patient's willingness to switch products. Of the responding physicians, $40 \%$ expressed no opinion regarding patient feedback about convenience receiving the prescription, while $48 \%$ of responding physicians expressed no opinion regarding patient perception of therapeutic response. Responding physicians slightly agreed that patient feedback had been positive regarding the patient's willingness to switch products.

\section{Solicit Physician's Opinion}

For the statement, "Practicing physicians' opinions should be solicited prior to implementing therapeutic conversion programs," a mean response of $8.280+2.227$ was generated. This suggests that responding physicians strongly agreed that the practicing physician's opinion should be solicited prior $10 \mathrm{im}$ plementing therapeutic conversion programs. Obviously, prac- titioners appreciate involvement in decisions surrounding issues of clinical importance.

Although the objective of this research was to evaluate the qualitative aspects of a therapeutic conversion program within the staff-model $\mathrm{HMO}$, quantitative findings show increased prescribing of preferred and alternative products from $20.6 \%$ to $69.2 \%$ within the HMG-CoA Reductase Inhibitor class from the close of Q1 1994 to the close of Q1 1995. This represents a $26.5 \%$ reduction in average ingredient costs over the corresponding 12 -month period.

\section{DISCUSSION AND CONCLUSION}

This evaluation indicated that responding HMO physicians were not displeased with the Lescol therapeutic conversion program. The program rationale was clear, the supporting communications were useful, the inconveniences were slight, the patient feedback was neutral, and, on average, the clinical concerns about Lescol were minor. The vast majority (96\%) of responding physicians expressed strong agreement that the practicing physician's opinion should be solicited before implementing therapeutic conversion programs.

Further, the generalizability of the findings generated from this study are limited to the one staff-model HMO under study. The salient concerns regarding a particular therapeutic conversion program are unique to an individual HMO setting and to the drugs involved. This is further limited by the type of HMO setting (staff-model versus IPA-model) under study and the quality of administrative and communication efforts within these settings. Given the strong response from this study that physicians' opinions should be solicited, it is recommended that the qualitative aspects of therapeutic conversion programs be assessed in other managed care settings to continually improve their future programs and facilitate collaborative relationships between pharmacists and physicians.

One of the physicians provided an insighıful written comment regarding managing health care costs: "Rather than be obsessed with single-line item costs such as pharmacy, we need to move toward total disease management, such as for coronary artery disease. Prescription cost is about $6 \%$ of the disease cost [within this staff-model HMO setting]. Putting great focus on the $6 \%$ while risking exacerbation of the $94 \%$ is literally penny-wise and dollar-foolish."

\footnotetext{
A References

1. Salmon JW. The professional and corporate context. In: Davis, R, ed. Contested ground: public purpose and private interest in the regulation of prescription drugs. London: Oxford University Press, 1996.

2. Salmon JW, White W, Feinglass J. The futures of physicians: agency and autonomy reconsidered. Theoret Med 1990; 11: 261-74.

3. Kellick KA, Burns K, McAndrew E, Haberl E, Hook N, Ellis A. Outcome monitoring of fluvastatin in a Department of Veterans Affairs lipid clinics. Am J Cardiol 1995; 76:62A-64A
} 